\title{
Use of FLT3 inhibitors in acute myeloid leukemia remission induction or salvage therapy: systematic review and meta-analysis
}

This article was published in the following Dove Press journal:

Cancer Management and Research

\author{
Minglei Yang* \\ Jian Zhao* \\ Tielong Liu \\ Xinghai Yang \\ Haifeng Wei \\ Wei Xu \\ Jianru Xiao
}

Department of Orthopedic Oncology, Changzheng Hospital, Second Military Medical University, Shanghai, 200003, China

*These authors contributed equally to this work
Correspondence: Wei Xu; Jianru Xiao Department of Orthopedic Oncology, Changzheng Hospital, Second Military Medical University, 415 Fengyang Road, Shanghai 200003, China

Tel +86 2I I37 6I27 8657;

$+862113701785283$

Fax +862181885634 $+862181885634$

Email xuweichangzheng@hotmail.com; jianruxiao83@163.com
Background: Previous studies showed that FLT3 inhibitors played an important role in acute myeloid leukemia (AML) therapy. However, discrepancies remain regarding the association between FLT3 inhibitors use and prognosis of AML patients in clinical trials.

Aim: The aim of this study was to evaluate the effect of FLT3 inhibitors on the treatment of AML in a systematic review and meta-analysis.

Materials and methods: PubMed, Embase, and Cochrane Library databases were searched for studies published before August 2017 that used FLT3 inhibitors in AML. Fixed- and randomeffect models were used, and between-study heterogeneity was assessed.

Results: A total of 26 studies fitting our inclusion and exclusion criteria were included. The FLT3 status of patients and main treatment outcomes including overall survival (OS), eventfree survival (EFS), relapse-free survival (RFS), complete remission (CR), and overall response rate (ORR) after therapy were extracted. Five studies comparing addition of FLT3 inhibitors and placebo or blank control to chemotherapy were analyzed in Part I, showing improved OS (hazard ratio $[\mathrm{HR}]=0.86,95 \%$ confidence interval $[\mathrm{CI}]=0.75-0.99, P=0.03$ ) in the FLT3 inhibitor group but without a significant improvement on $\mathrm{EFS}(\mathrm{HR}=0.86,95 \% \mathrm{CI}=0.62-1.21, P=0.39)$ and ORR (odds ratio $[\mathrm{OR}]=1.10,95 \% \mathrm{CI}=0.89-1.35, P=0.38$ ). Twenty-one studies evaluating the benefit of using FLT3 inhibitors in different FLT3-type AML patients were analyzed in Part II, showing that FLT3-internal tandem duplication (ITD)-positive patients were more sensitive to FLT3 inhibitor treatment and achieved better $\mathrm{CR}(\mathrm{OR}=1.89,95 \% \mathrm{CI}=1.06-3.37, P=0.03)$ and ORR (OR=3.07, 95\% CI=2.13-4.43, $P<0.001$ ).

Conclusion: Our study showed that combined use of FLT3 inhibitors improved OS and that the FLT3 status of AML patients could affect their sensitivity to FLT3 inhibitors in terms of CR and ORR.

Keywords: acute myeloid leukemia, FLT3-ITD, clinical outcome, meta-analysis

\section{Introduction}

Acute myeloid leukemia (AML) is a heterogeneous hematologic malignancy characterized by clonal expansion of myeloid blasts in the peripheral blood, bone marrow (BM), and/or other tissues, with an incidence of 3.7/100,000 individuals. ${ }^{1,2}$ Patients with AML are divided into different risk states based on their cytogenetic and molecular abnormalities. FLT3 protein is a member of the receptor-tyrosine kinase (RTK) family, playing an important role in normal growth and differentiation of hematopoietic precursor cells. ${ }^{3,4}$ In currently available studies, ${ }^{4,5}$ the internal tandem duplications (ITDs) in FLT3 mutations were related to the blast of leukemia cells 
and suppression of apoptosis and were detected in $\sim 23 \%$ AML patients. A meta-analysis ${ }^{6}$ of AML patients showed that the pooled hazard ratio (HR) of the impact of FLT3-ITD on overall survival (OS) and relapse-free survival (RFS) was $1.86(95 \%$ confidence interval $[\mathrm{CI}]=1.57-2.20)$ and 1.83 (95\% CI=1.54-2.18), respectively. The goal of treatment of AML is to achieve morphologic, cytogenetic, and molecular complete remission (CR); prepare for subsequent consolidation chemotherapy or hematopoietic cell transplantation (HCT); and get long-term OS, event-free survival (EFS), and RFS. ${ }^{2}$ Patients with FLT3-ITD mutations are considered to a have a higher risk of relapse with shorter RFS, EFS, and OS and are recommended for clinical trials where available. ${ }^{5,7}$

FLT3 inhibitors are a type of tyrosine kinase inhibitors with FLT3 inhibitory activity. Preclinical research and clinical trials ${ }^{8,9}$ have confirmed the activity of FLT3 inhibitors against AML primary blasts with FLT3-ITD mutations in vitro and in vivo. Current studies ${ }^{8,10}$ have demonstrated that the patients can benefit from the use of FLT3 inhibitors. Sunitinib, midostaurin, lestaurtinib, sorafenib, quizartinib, and crenolanib are the most common FLT3 inhibitors reported in current clinical trials. Most of these drugs were first approved for use in solid malignancies by the US Food and Drug Administration (FDA). Except for several Phase I and Phase II clinical trials on the safety and effectiveness of these compounds, ${ }^{11-31}$ there have been limited studies to evaluate the dosage, duration, monotherapy, or combination use with chemotherapy of these drugs in AML. ${ }^{32}$ In addition, the demographic baselines in these studies varied substantially. Only a few randomized controlled trials (RCTs) or Phase III clinical trials ${ }^{33-37}$ have compared midostaurin-, lestaurtinib-, or sorafenib-containing new regimens with the standard chemotherapy regimens.

There is no consensus on the role of FLT3 inhibitors in AML patients. The outcomes of clinical trials are controversial, and high risks of adverse events are also associated with these agents. ${ }^{33}$ Knowing that there is no systematic review and meta-analysis focusing on the role of FLT3 inhibitors in AML therapy, we conducted this meta-analysis in an attempt to clarify the clinical benefit of FLT3 inhibitors and make a summary on the clinical outcomes in terms of CR, overall response rate (ORR), OS, and EFS when FLT3 inhibitors are added to conventional chemotherapy by comparing them with chemotherapy alone (Part I). We also compared CR or ORR between AML patients with different FLT3-ITD statuses when FLT3 inhibitors were used (Part II).

\section{Materials and methods}

\section{Search strategy}

The following literature searching strategy was used to identify potentially relevant studies focusing on FLT3 inhibitors in AML induction/salvage therapy. PubMed, Embase, and Cochrane Library databases were searched by using the following index terms: leukemia, myeloid, acute AND FLT3 OR tyrosine kinase inhibitor OR sorafenib OR sunitinib OR midostaurin OR lestaurtinib OR quizartinib OR crenolanib, without restrictions on the region and publication type. Mesh subject words with random words were selected from the PubMed database. No language limit was required for publication. Relevant reference lists were screened through manual search for further potential studies. When multiple publications reported on the same study population, the report that was most complete or had the longest follow-up period was used. The last date of the search was August 3, 2017 (Figure 1).

\section{Study selection}

Two reviewers independently reviewed all studies and selected eligible trials. To analyze the potential benefit from adding FLT3 inhibitors to AML treatment in mixed population, we used the following inclusion criteria in Part I: 1) RCTs involving comparison between FLT3 inhibitors and placebo or blank control, with or without other chemotherapy compounds in AML remission induction and/or salvage treatment; 2) studies using CR, morphologic CR with incomplete blood count recovery (CRi), morphologic CR with incomplete platelet recovery (CRp), partial remission (PR), OS, RFS, and EFS as the response criteria; and 3) the definition of the above response criteria in accordance with the recommendations of the International Working Group. ${ }^{38}$ CR was defined by the presence of $<5 \%$ blasts in the BM with $1 \times 10^{9} / \mathrm{L}$ neutrophils and $100 \times 10^{9} / \mathrm{L}$ platelets in the peripheral blood. CRp was defined in patients with CR but persistent platelet count $<100 \times 10^{9} / \mathrm{L}$. CRi was defined in patients with persistent neutrophil count $<1 \times 10^{9} / \mathrm{L}$ or without platelet recovery. PR required all hematologic values for a $\mathrm{CR}$ but with a decrease of at least $50 \%$ in the percentage of blasts to $5-25 \%$ in the BM aspirate. To analyze the possible effect of FLT3 inhibitors on AML with different FLT3 statuses, we used the following inclusion criteria in Part II: 1) clinical trials involving the outcome of using FLT3 inhibitors for the treatment of AML; 2) studies using CR, CRi, CRp, PR, OS, RFS, and EFS as the response criteria; 3) patients involving gene analysis and treatment outcomes stratified on different 


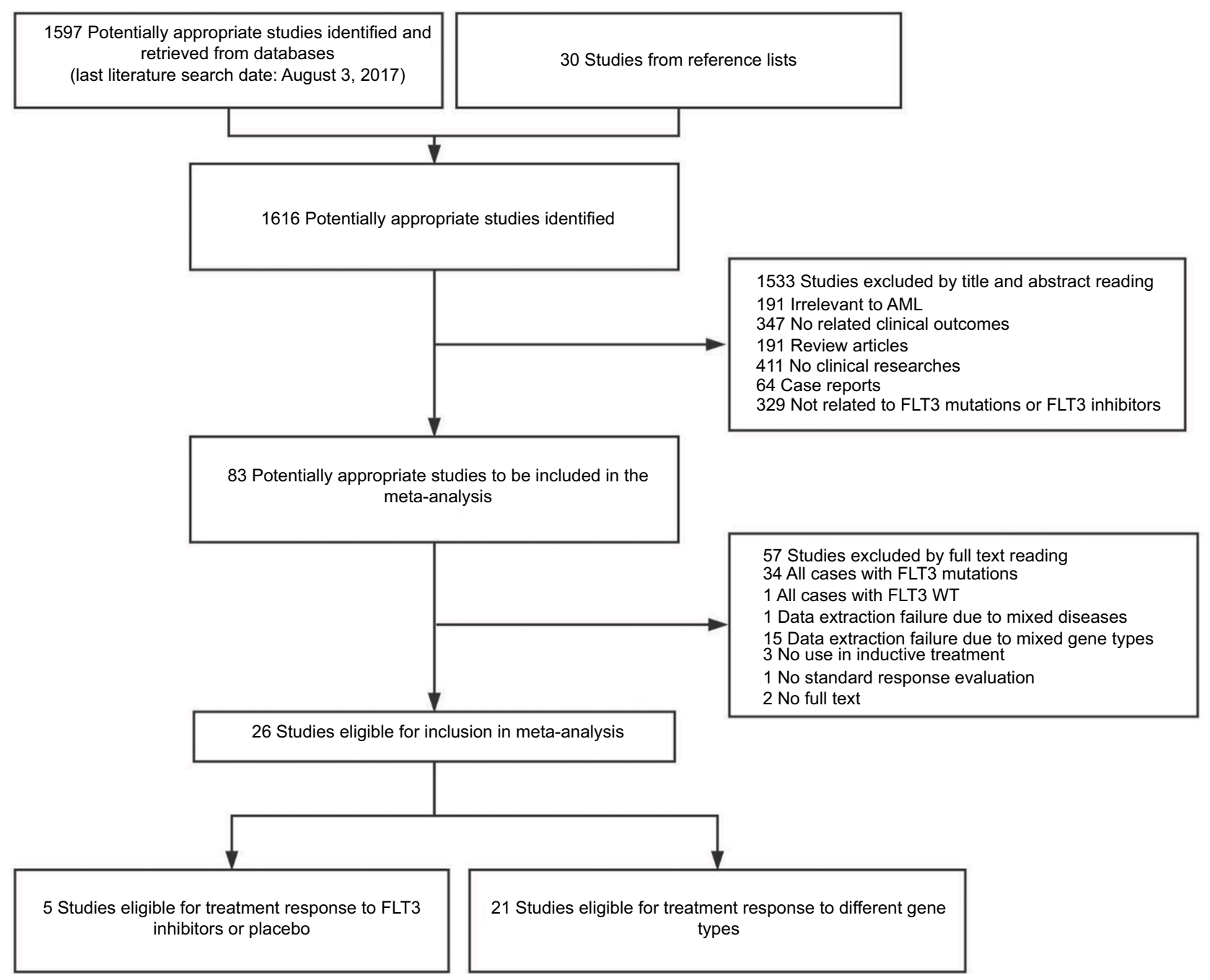

Figure I Flow diagram concerning study identification, inclusion, and exclusion.

Abbreviations: AML, acute myeloid leukemia; FLT3, FMS-like tyrosine kinase 3; WT, wild type; NO, without.

FLT3 status; and 4) studies involving patients with multiple types of FLT3 status. Owing to high AML transformation in myelodysplastic syndrome (MDS) and related therapeutic regimens between AML and MDS, studies with fewer than $10 \%$ MDS patients were included in our analysis. Studies that could not meet any one of the above inclusion criteria were excluded. Animal studies, review articles, and case reports were also excluded.

\section{Data extraction}

Two reviewers independently extracted the data from the full manuscripts of included articles, and any disagreement was resolved by the third reviewer. We obtained the following information from each article: first author, year of publication, number of included patients, gene type of patients, therapeutic regimen, prior therapeutic regimen, type of AML, AML status, and treatment outcome. The main treatment outcomes included CR, CRi, CRp, PR, OS, RFS, and EFS after AML induction or salvage therapy in different regimens (in Part I) or in different FLT3 status patients (in Part II). Patients with the therapy outcome better than or equal to PR were thought to respond to the current treatment regimen and calculated for ORR. The quality of studies was assessed by using the Cochrane risk of bias assessment tool for RCTs (Figure S1) and Newcastle-Ottawa Quality Assessment Scale (NOS) for cohort studies (Tables 1 and S1).

\section{Statistical analysis}

This meta-analysis was performed with the aid of software Review Manager Version 5.3 (available at http://ims.cochrane. org/revman/download). The number of main outcome events and simple size were calculated. In the forest plots, odds ratio (OR), HR, and their $95 \% \mathrm{CI}$ were displayed in our qualitative data synthesis. To assess the heterogeneity between studies, 


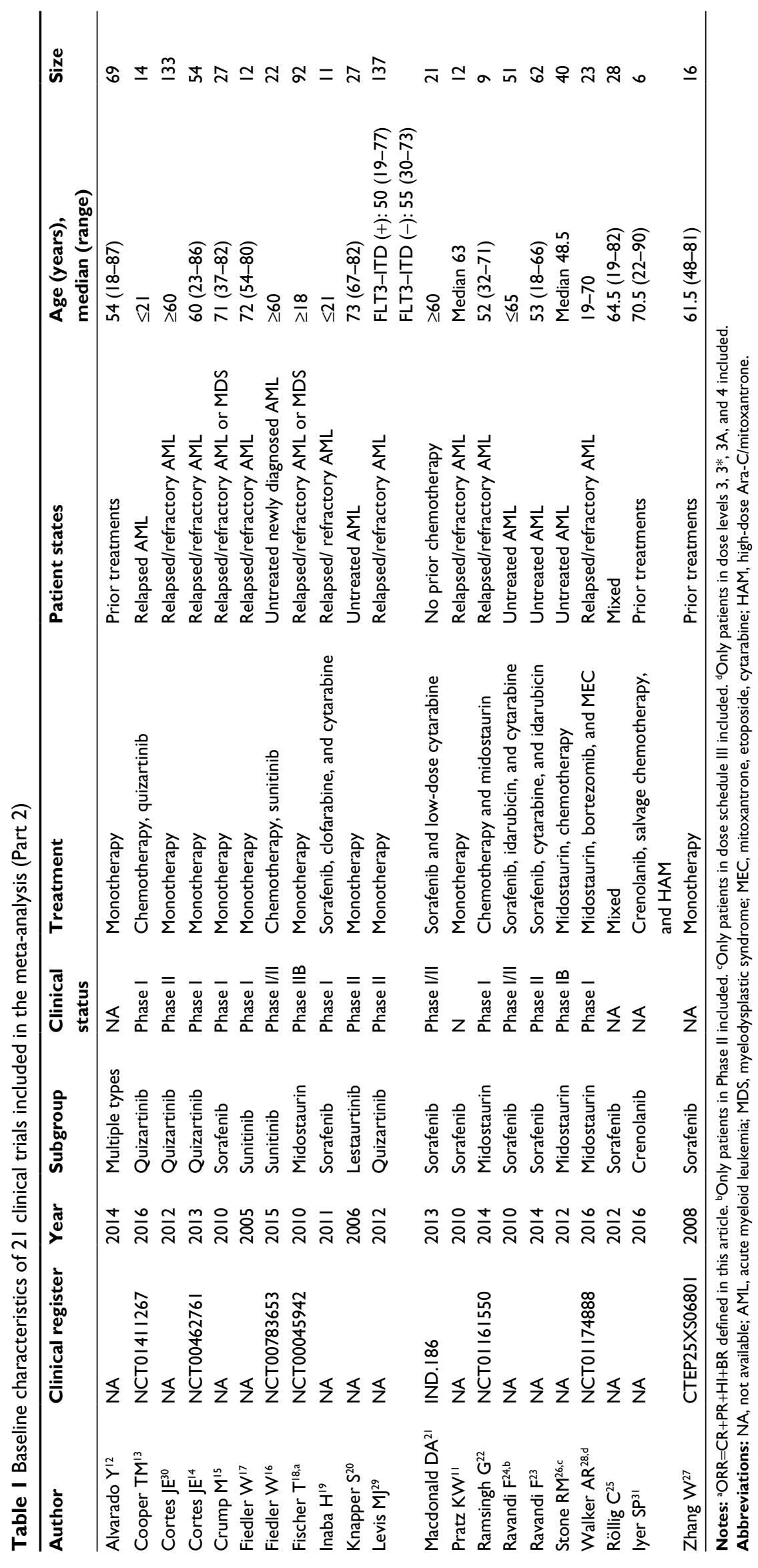


the Cochran's $Q$ test and $I^{2}$ test were used. If the Cochran's $Q$ test showed $P<0.05$ or the $I^{2}$ test exhibited $P>50 \%$, indicating significant heterogeneity between studies, the random-effect model would be used. If the heterogeneity was acceptable, the fixed-effect model would be used. Subgroup analyses on different types of FLT3 inhibitors were performed to further evaluate the heterogeneity and sensitivity. Funnel plots were used to analyze the publication bias.

\section{Results}

\section{Study characteristics}

A total of 26 studies were identified and included in our study. Five RCTs providing the data about addition of FLT3 inhibitor versus placebo or blank control to conventional chemotherapy in AML were analyzed in Part I. The other 21 articles were analyzed in Part II to evaluate the benefit of using FLT3 inhibitors between AML patients with and without FLT3-ITD mutations. There were 1905 patients involved in Part I and 866 patients involved in Part II. Features of the included studies are summarized in Tables 1 and 2. In Part I, two studies ${ }^{33,36}$ used sorafenib in 464 patients, two ${ }^{34,35}$ used lestaurtinib in 724 patients, and one ${ }^{39}$ used midostaurin in 717 patients. Four studies ${ }^{33,34,36,37}$ selected untreated AML, and the other ${ }^{35}$ involved patients with relapsed AML. In Part II, eight studies ${ }^{11,15,19,21,23-25,27}$ used sorafenib, four ${ }^{13,14,29,30}$ used quizartinib, four ${ }^{18,22,26,28}$ used midostaurin, two ${ }^{16,17}$ used sunitinib, one ${ }^{20}$ used lestaurtinib, one ${ }^{31}$ used crenolanib, and one ${ }^{12}$ used multiple types of FLT3 inhibitors. In one study, ${ }^{18}$ ORR included not only $\mathrm{CR}, \mathrm{CRi}, \mathrm{CRp}$, and PR but also hematologic improvement (HI) and blast response (BR).

\section{Part I: effects of adding FLT3 inhibitors to conventional chemotherapy}

In five studies included in this part, the end points including OS, EFS, and ORR (containing CR, CRp, and CRi) were analyzed for assessing the clinical effect. HR of OS from four articles (two sorafenib, one lestaurtinib, and one midostaurin) was pooled with no significant heterogeneity between them $\left(P=0.56, I^{2}=0 \%\right)$. Fixed-effect models were selected, and a forest plot was performed to show the results $(\mathrm{HR}=0.86,95 \% \mathrm{CI}=0.75-0.99, P=0.03$; Figure 2). HR of EFS from three articles (two sorafenib and one midostaurin) was pooled using random-effect models $(\mathrm{HR}=0.86,95 \% \mathrm{CI}=0.62-1.21, P=0.39)$, with heterogeneity between studies $\left(P=0.006, I^{2}=80 \%\right.$; Figure 3$)$. We also pooled the ORR from five articles with no significant heterogeneity $\left(P=0.41, I^{2}=0 \%\right)$ and showed the result $(\mathrm{OR}=1.10,95 \% \mathrm{CI}=0.89-1.35, P=0.38)$ using fixed-effect

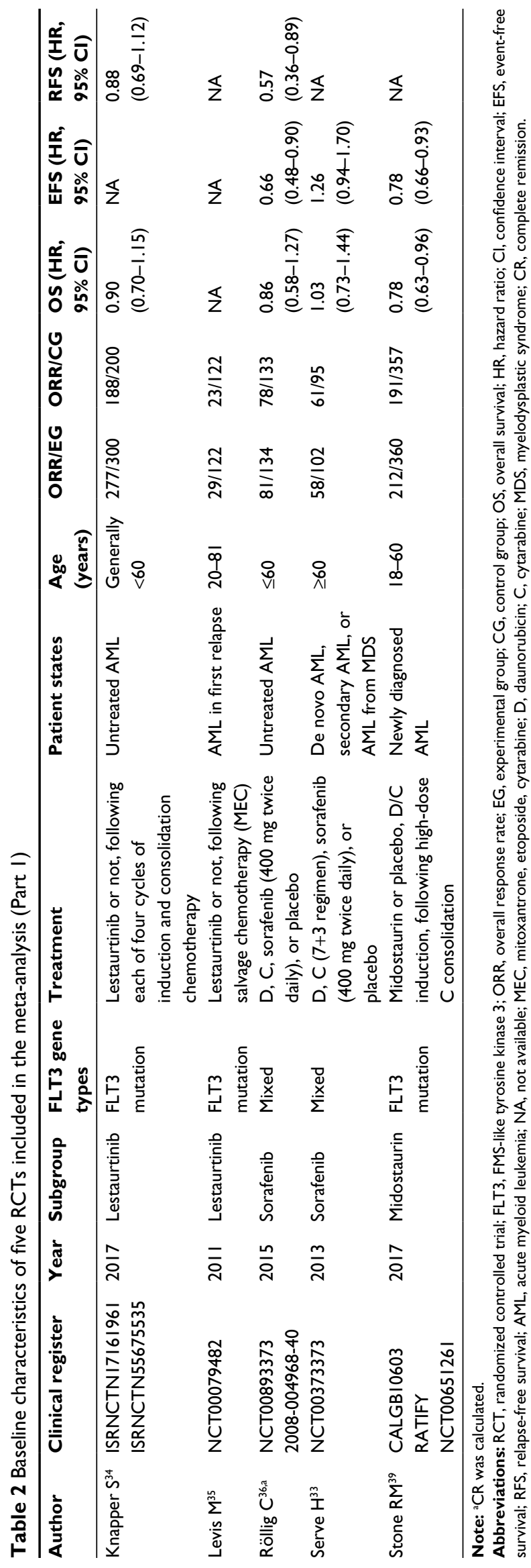




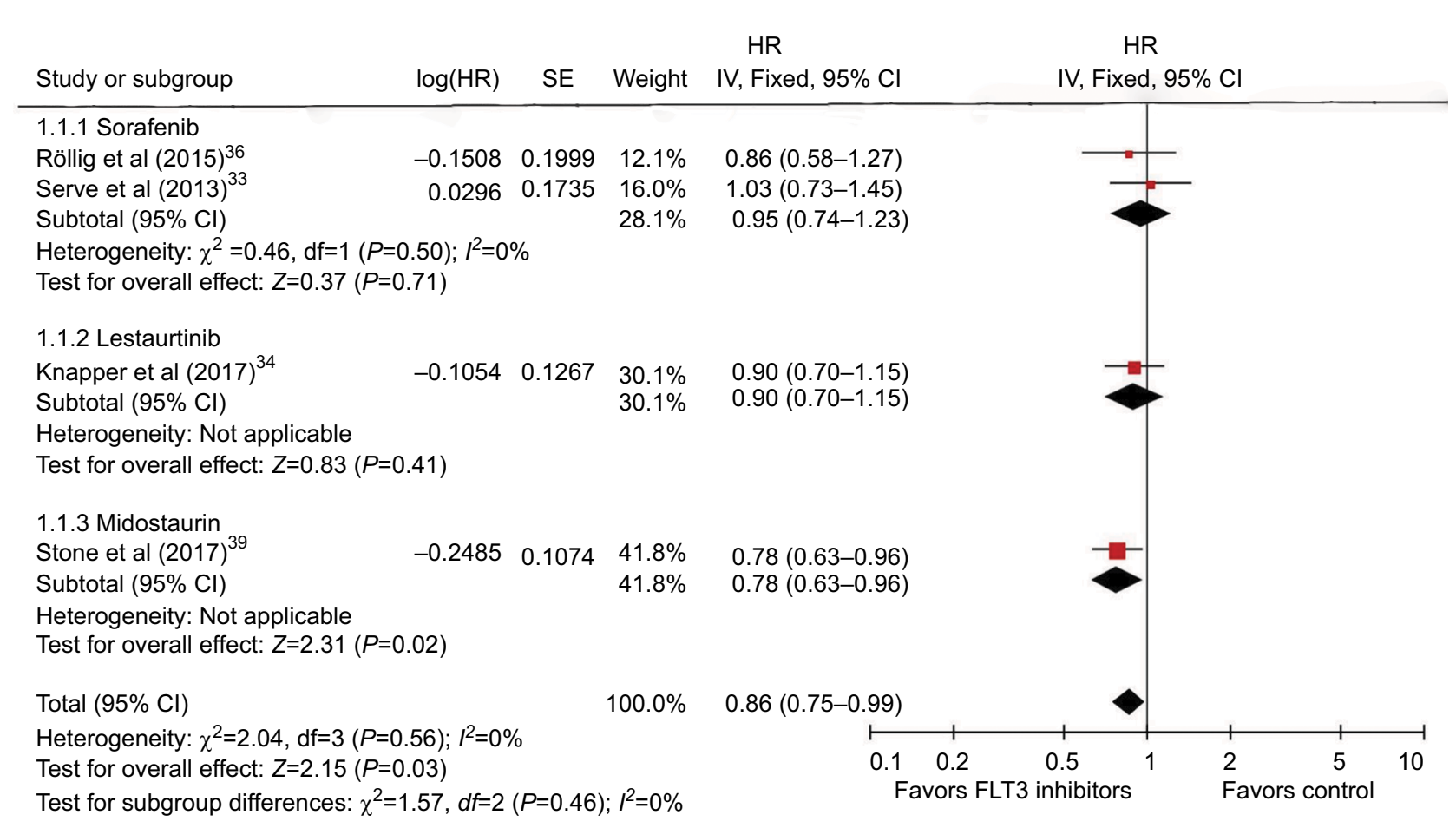

Figure 2 Forest plot for pooled HR of OS between combined use of FLT3 inhibitors with chemotherapy and chemotherapy alone in patients with AML. Abbreviations: HR, hazard ratio; OS, overall survival; FLT3, FMS-like tyrosine kinase 3; AML, acute myeloid leukemia; SE, standard error; IV, inverse variance; Cl, confidence interval.

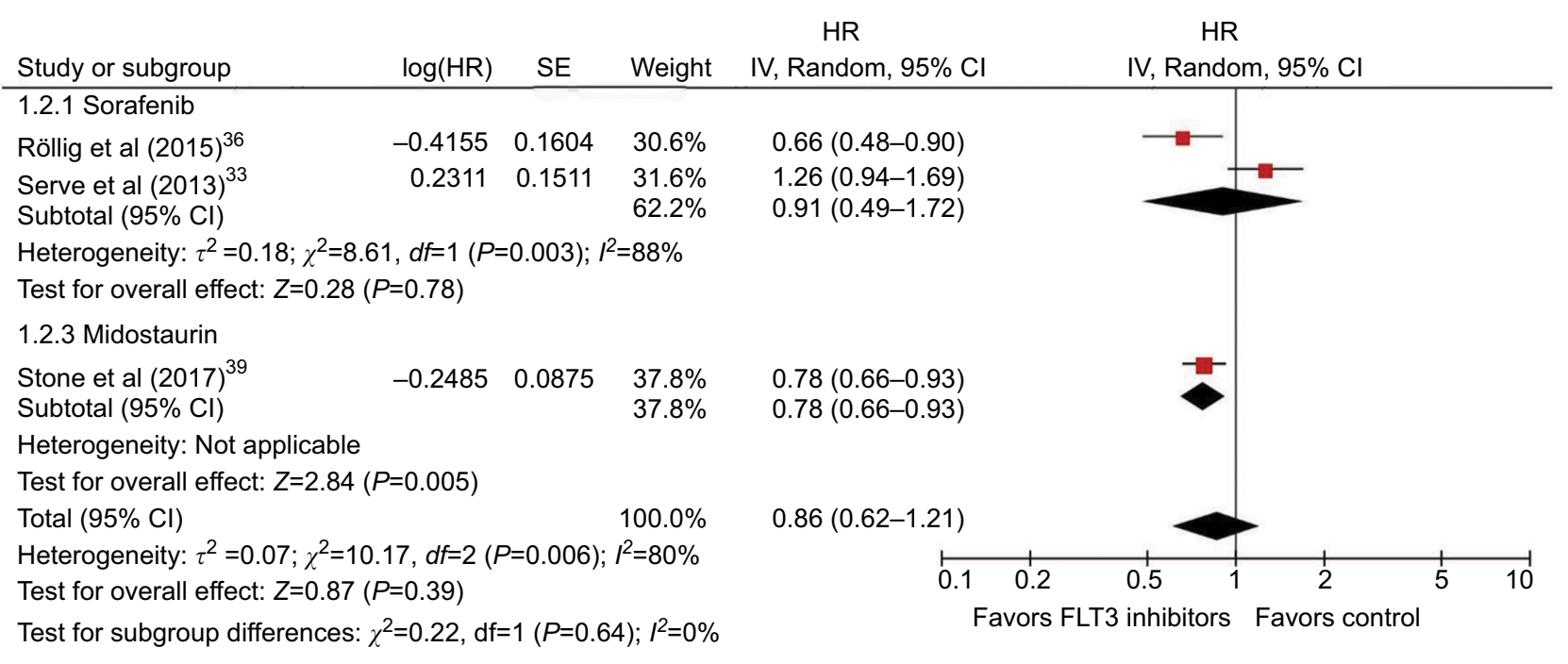

Figure 3 Forest plot for pooled HR of EFS between combined use of FLT3 inhibitors with chemotherapy and chemotherapy alone in patients with AML.

Abbreviations: HR, hazard ratio; EFS, event-free survival; FLT3, FMS-like tyrosine kinase 3; AML, acute myeloid leukemia; SE, standard error; IV, inverse variance; Cl, confidence interval.

models (Figure 4). The pooled results showed improved OS with the addition of FLT3 inhibitors to conventional chemotherapy, while no significant improvement on EFS and ORR was seen.

\section{Part I: sensitivity and subgroup analysis}

When we changed the fixed-effect models to randomeffect models for pooled analysis of the HR of OS, the results remained the same $(\mathrm{HR}=0.86,95 \% \mathrm{CI}=0.75-0.99$, 


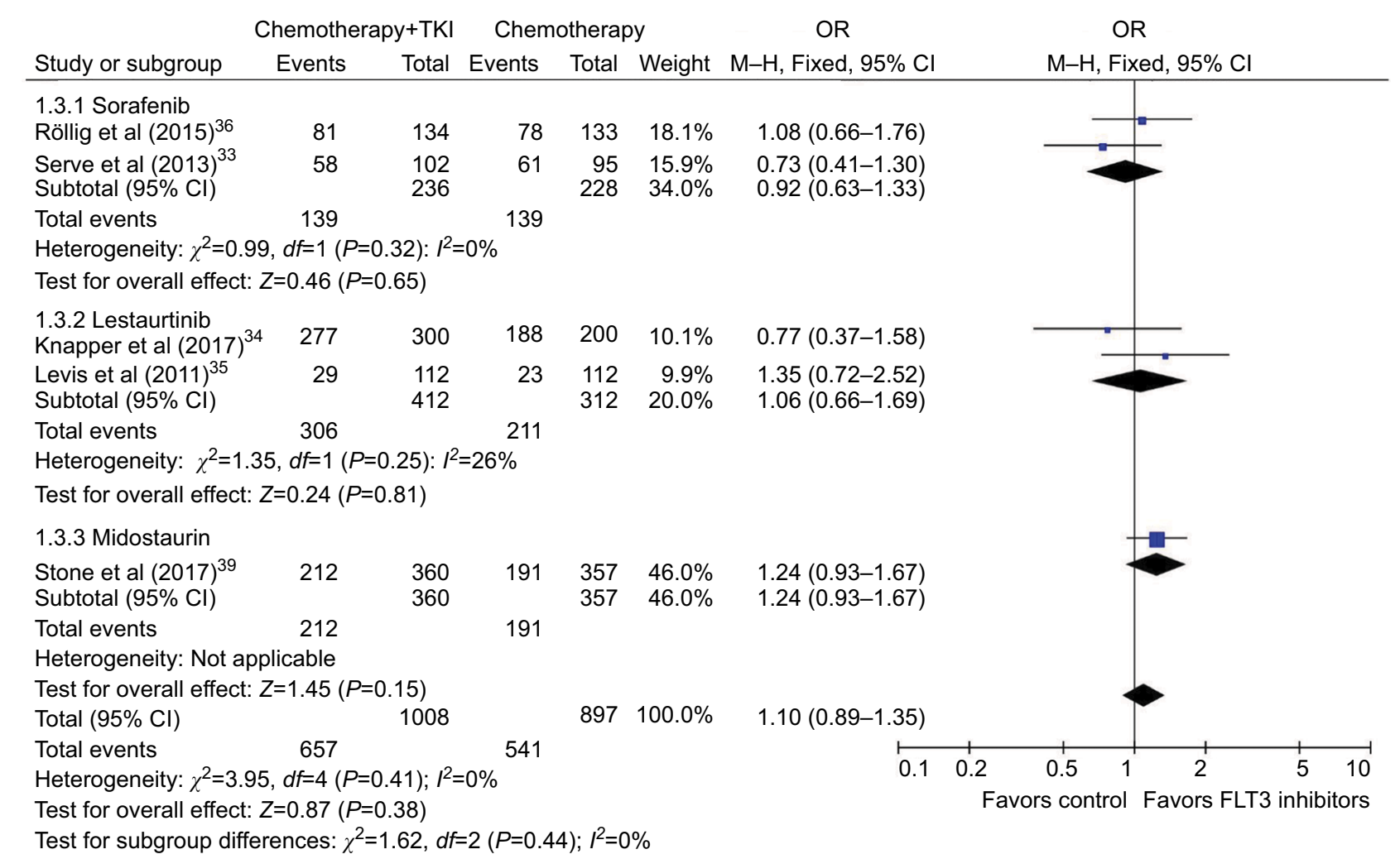

Figure 4 Forest plot for pooled OR of ORR between combined use of FLT3 inhibitors with chemotherapy and chemotherapy alone in patients with AML. Abbreviations: OR, odds ratio; ORR, overall response rate; FLT3, FMS-like tyrosine kinase 3; AML, acute myeloid leukemia; TKI, tyrosine kinase inhibitor; M-H, MantelHaenszel; $\mathrm{Cl}$, confidence interval

$P=0.03$ ); when we changed to use fixed-effect models for pooled analysis of the HR of EFS, the result showed a significant improvement in EFS in the combined therapy group ( $\mathrm{HR}=0.83,95 \% \mathrm{CI}=0.73-0.95, P=0.008)$. Two sorafenib studies included patients with different FLT3 statuses, and a sensitivity analysis on ORR was conducted using the data based on FLT3-ITD patients (data from SORAML study were not changed because no details were extracted), also showing no significant improvement on ORR (OR=1.14, 95\% $\mathrm{CI}=0.92-1.43, P=0.23$; heterogeneity $P=0.55, I^{2}=0 \%$ ). The pooled data of subgroup analysis by types of FLT 3 inhibitors showed no significant improvement in ORR in the sorafenib subgroup as compared with the current conventional chemotherapy $(\mathrm{OR}=0.92,95 \% \mathrm{CI}=0.63-1.33, P=0.65$; heterogeneity $P=0.32, I^{2}=0 \%$ ). In the lestaurtinib subgroup, the pooled ORR $(\mathrm{OR}=1.06,95 \% \mathrm{CI}=0.66-1.69, P=0.81$; heterogeneity $P=0.25, I^{2}=26 \%$ ) was also not statistically significant. When we changed the statistical methods to random-effect models or used pool risk ratio (RR), the results were consistent.

\section{Part II: effect of FLT3 inhibitors between FLT3-ITD-positive and -negative AML}

A total of 21 studies were included in Part II for studying the different efficacies of FLT3 inhibitors on FLT3-ITD or
non-FLT3-ITD AML. We were unable to extract the CR data in one of the articles. ${ }^{18}$ In all, 20 studies were pooled to analyze CR and 21 studies were pooled to analyze ORR. In CR analysis, 397 patients with FLT3-ITD and 377 patients without this mutation were included. There was no significant heterogeneity $\left(P=0.97, I^{2}=0 \%\right)$. Fixed-effect models were selected, and a forest plot was performed to show the results $(\mathrm{OR}=1.89,95 \% \mathrm{CI}=1.06-3.37, P=0.03$; Figure 5$)$. The pooled result showed better performance on CR when FLT3 inhibitors were used in FLT3-ITD-positive AML patients compared to FLT3-ITD-negative patients.

Then, we pooled the ORR from all 21 articles. There were 423 patients with FLT3-ITD mutation and 443 patients without this mutation. The heterogeneity $\left(P=0.69, I^{2}=0 \%\right)$ was not statistically significant, and fixed-effect models were selected. A forest plot was performed to show the results $(\mathrm{OR}=3.07$, 95\% CI $=2.13-4.43, P<0.001$; Figure 6). Our finding showed that FLT3-ITD-positive AML patients were more sensitive to FLT3 inhibitors, benefited more from FLT3 inhibitor treatment and therefore could get better ORR.

\section{Part II: sensitivity and subgroup analysis}

Studies about sorafenib, quizartinib, and midostaurin were selected for subgroup analysis. In the sorafenib subgroup, 


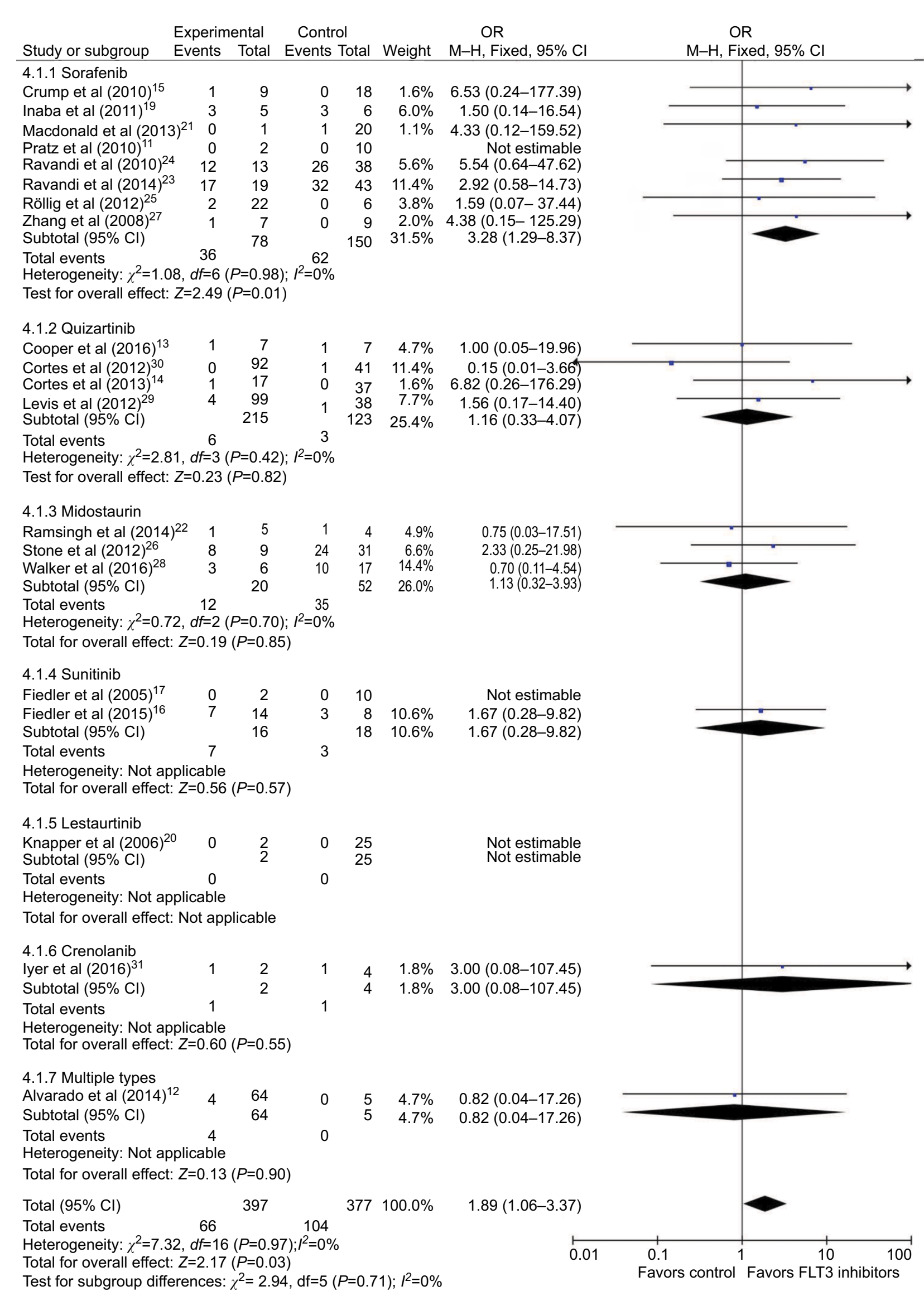

Figure 5 Forest plot for pooled OR of CR between FLT3-ITD-positive patients and FLT3-ITD-negative patients.

Abbreviations: OR, odds ratio; CR, complete remission; FLT3, FMS-like tyrosine kinase 3; ITD, internal tandem duplication; M-H, Mantel-Haenszel; Cl, confidence interval. 


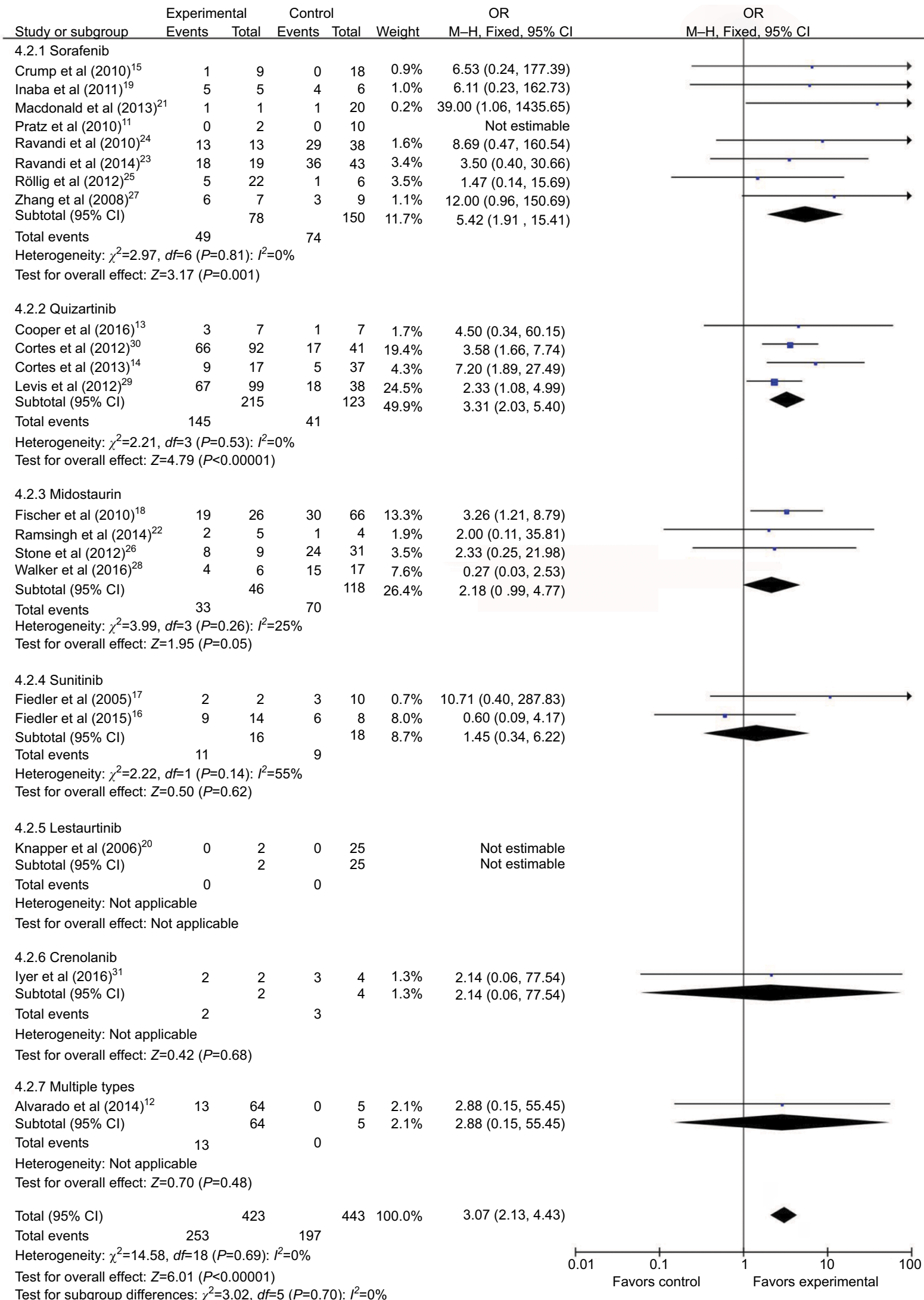

Figure 6 Forest plot for pooled OR of ORR between FLT3-ITD-positive patients and FLT3-ITD-negative patients.

Abbreviations: OR, odds ratio; ORR, overall response rate; $\mathrm{FLT3}$, FMS-like tyrosine kinase 3; ITD, internal tandem duplication; $\mathrm{M}-\mathrm{H}$, Mantel-Haenszel; $\mathrm{Cl}$, confidence interval. 
eight relevant studies were pooled, showing better $\mathrm{CR}$ $(\mathrm{OR}=3.28,95 \% \mathrm{CI}=1.29-8.37, P=0.01$; heterogeneity $\left.P=0.98, I^{2}=0 \%\right)$ and ORR $(\mathrm{OR}=5.42,95 \% \mathrm{CI}=1.91-15.41$, $P=0.001$; heterogeneity $P=0.81, I^{2}=0 \%$ ) in patients with FLT3-ITD-positive AML. In the quizartinib subgroup, four relevant studies were pooled, showing that patients with FLT3-ITD-positive AML got better ORR (OR=3.31, 95\% $\mathrm{CI}=2.03-5.40, P<0.001$; heterogeneity $P=0.53, I^{2}=0 \%$ ). Quizartinib did not seem to improve CR significantly $(\mathrm{OR}=1.16,95 \% \mathrm{CI}=0.33-4.07, P=0.82$; heterogeneity $\left.P=0.42, I^{2}=0 \%\right)$ in FLT3-ITD-positive AML patients. No significant preference of $\mathrm{CR}(\mathrm{OR}=1.13,95 \% \mathrm{CI}=0.32-3.93$, $P=0.85$; heterogeneity $\left.P=0.70, I^{2}=0 \%\right)$ and $\mathrm{ORR}(\mathrm{OR}=2.18$, 95\% CI=0.99-4.77, $P=0.05$; heterogeneity $P=0.26, I^{2}=25 \%$ ) in FLT3-ITD-positive AML was observed in the midostaurin subgroup (Figures 5 and 6). Subgroup analysis was also carried out based on primary or relapsed AML. In the primary AML subgroup, six relevant studies were pooled, showing better $\mathrm{CR}(\mathrm{OR}=2.90,95 \% \mathrm{CI}=1.17-7.18, P=0.02$; heterogeneity $\left.P=0.94, I^{2}=0 \%\right)$ and ORR $(\mathrm{OR}=2.78,95 \%$ $\mathrm{CI}=1.06-7.32, P=0.04$; heterogeneity $P=0.28, I^{2}=22 \%$ ) in FLT3-ITD-positive patients. In the relapsed AML subgroup, 15 studies were included and FLT3-ITD-positive patients had better ORR $(\mathrm{OR}=3.13,95 \% \mathrm{CI}=2.11-4.65, P<0.001$; heterogeneity $P=0.74, I^{2}=0 \%$ ), whereas no significant preference of $\mathrm{CR}(\mathrm{OR}=1.34,95 \% \mathrm{CI}=0.63-2.88, P=0.45$; heterogeneity $P=0.93, I^{2}=0 \%$ ) was detected. In all, 14 studies with $>20$ participants included were selected for sensitivity analysis; the results remained the same in either $\mathrm{CR}(\mathrm{OR}=1.96,95 \%$ $\mathrm{CI}=1.03-3.72, P=0.04$; heterogeneity $P=0.84, I^{2}=0 \%$ ) or ORR (OR=2.88, 95\% CI=1.96-4.24, $P<0.001$; heterogeneity $P=0.41, I^{2}=4 \%$ ).

\section{Publication bias}

Because only five studies were included in Part I, funnel plots had low power and were not used in test for publication bias. In Part II, we obtained symmetric funnel plots for the synthesis of CR and ORR, indicating mild publication bias (Figure S2A and B).

\section{Discussion}

AML is a hematologic malignancy characterized by multiple cytogenetic and genetic abnormalities in the hematopoietic stem or progenitor cells. ${ }^{5}$ As a common mutation, FLT3-ITD was detected in $20 \%-30 \%$ of AML patients and reported to be associated with worse prognosis. ${ }^{40}$ Patients with FLT3-ITD mutations and a normal karyotype are considered in the poorrisk group according to the National Comprehensive Cancer
Network (NCCN) guidelines. In the genetic risk classification of the 2017 European LeukemiaNet (ELN) recommendation, a high FLT3-ITD allelic ratio is thought to be associated with a high relapse rate and poor OS. Patients with NPM1 mutation and FLT3-ITD with a low allelic ratio belong to the favorable risk group, while AML patients with wild-type NPM1 and FLT3-ITD with a high allelic ratio have a poor prognosis and are placed in the adverse-risk group. ${ }^{41,42}$ FLT3 inhibitors have been applied to target mutant FLT3 and block related pathways potentially involved in the development and progression of malignancies. ${ }^{43}$ However, there is no consensus on the use of FLT3 inhibitors, and most of these compounds are "off-label" used in AML.

FLT3 inhibitors undergoing clinical evaluation were divided into the first-generation agents (including sunitinib, midostaurin, and lestaurtinib) with relative nonspecificity and multiple targets and the second-generation agents (including sorafenib, quizartinib, and crenolanib) with a higher selectivity against mutant FLT3. ${ }^{32} \mathrm{~A}$ host of first-generation FLT3 inhibitors were limited by their suboptimal propensity for AML control in vivo or their off-target effects, frequently producing a peripheral blast cell clearance and BM blast cell persistence. ${ }^{10,44}$ In our study, no patient was found to achieve ORR with lestaurtinib monotherapy and $41.7 \%$ patients responded to sunitinib monotherapy with the best outcome of PR. ${ }^{17,20}$ It was documented that midostaurin as a single agent could reduce blast count in peripheral blood in $70 \%$ patients, and the peripheral blood and BM BR was shown in $53.3 \%$ patients with monotherapy in studies from our analysis. ${ }^{18,45}$ For second-generation FLT3 inhibitors, quizartinib monotherapy has been reported with composite CR (CRc; including CR, CRi, and CRp) in 46-57\% patients with relapsed/refractory (R/R) AML. ${ }^{14,46,47}$ Crenolanib, as a single agent, achieved the CRc up to $39 \%$ in this patient population. ${ }^{48-50}$ The median OS for quizartinib and crenolanib in initial clinical studies was reported up to 168 and 259 days, and median EFS could achieve 74 and 56 days, respectively, which is similar or better than conventional chemotherapy with median OS of 198 days and 4-month EFS ranging from $16.6 \%$ to $37.7 \% .{ }^{50-52}$ Improvement on EFS and RFS has also been seen from the use of sorafenib. ${ }^{36}$ In the studies included in our analysis, $>50 \%$ the patients using sorafenib and quizartinib achieved ORR, and CR in two subgroups was $43.0 \%$ and $2.66 \%$, respectively, in FLT3-ITD-positive and -negative patients. The reason of much higher CR in the sorafenib subgroup might be that $75.9 \%$ (173/228) patients in the sorafenib subgroup received combined therapy regimen, whereas $95.9 \%$ (324/338) patients used quizartinib as a 
single agent. These results demonstrate notable and promising clinical efficacy of second-generation FLT3 inhibitors.

Compared with conventional chemotherapy, addition of FLT3 inhibitors seems to improve the OS of AML patients, although no significantly improved EFS and ORR were detected. When subgroup analysis was carried out on different FLT3 inhibitors, midostaurin was the only agent with a significantly higher OS and EFS, based on the results of CALGB 10603/RATIFY study. ${ }^{37}$ Midostaurin became the first FLT3 inhibitor for the treatment of FLT3-mutant AML, approved by the FDA on April 28, 2017 and the European Medicines Agency (EMA) on July 20, 2017. The results of the SORAML study ${ }^{36}$ showed that EFS and RFS were prolonged when sorafenib was added to standard chemotherapy in patients aged 60 years or younger with newly diagnosed AML, suggesting that sorafenib might have the potential to reduce relapses and increase the time in CR. However, another sorafenib study by Serve et $\mathrm{al}^{33}$ reported different results, which might be attributed to older age ( $>60$ years) of the participants. Considering that FLT3-ITD-positive patients might be more sensitive to these agents, the benefit of sorafenib on AML might be underestimated in these two studies, because both FLT3-mutated and FLT3-WT patients were included and FLT3-ITD-positive patients were a small part of all participants. No improved ORR was found in the pooled data or in each subgroup. It was documented that $\sim 50-80 \%$ primary AML patients and 18-60\% R/R AML patients could respond to conventional chemotherapy. ${ }^{53}$ The pooled ORR of combined use of sorafenib, lestaurtinib, and midostaurin in our analysis was $58.9 \%, 74.3 \%$, and $58.9 \%$, respectively, which might be influenced by the AML status of participants, and these results were consistent with the data of previous conventional chemotherapy. From the results of our analysis, FLT3 inhibitors seem to improve the OS of AML.

FLT3-ITD-positive patients might be more sensitive to FLT3 inhibitors with a higher CR and ORR compared to patients with other mutations or wild type when FLT3 inhibitors were used as combined therapy or monotherapy. This result could be explained by the block of FLT3-ITD-related oncogenic targets and signaling, leading to the suppression of leukemia blast cells. ${ }^{10}$ It has been documented that with conventional chemotherapy regimens, FLT3-ITD leads to a decreased disease-free survival (DFS) and OS, although the CR is comparable to that achieved in those with FLT3$\mathrm{WT}^{24,43}$ To clarify the potential long-term benefit of FLT3 inhibitors between FLT3-ITD-positive and -negative patients, the end points OS and DFS were reviewed and summarized from seven included studies (including three on midostaurin, three on quizartinib and one on sorafenib) where survival data on different gene types could be extracted. ${ }^{14,18,22,23,26,29,30}$ However, the quantitative analysis is inappropriate due to their various survival data forms. A Phase IIB trial of midostaurin $^{18}$ showed similar results to conventional chemotherapy, where the mutant population was with a median OS of 100 days ( $95 \% \mathrm{CI}=76-121$ days), shorter than that in wild-type population of 159 days (95\% CI=130-209 days), and it was the only study that provided statistical difference. A Phase II sorafenib clinical trial ${ }^{23}$ showed that the median OS and DFS for patients with FLT3-ITD was 15.5 and 9.9 months, respectively, shorter than for those without FLT3-ITD. Additional two studies on midostaurin reported the similar result that DFS and OS probabilities in patients with mutation were similar to those with FLT3-WT disease. ${ }^{22,26}$ Inconsistent results that whether FLT3-ITD-positive or -negative patients would have better OS and DFS were shown in quizartinib. ${ }^{14,29,30}$ For crenolanib, Cortes et $\mathrm{al}^{48}$ and Randhawa et $\mathrm{al}^{49}$ reported that the OS in mutant patients was longer than that in patients with negative FLT3-ITD, which might be due to the possible increased likelihood of patients able to undergo an allogeneic $\mathrm{HCT}^{36}$ In contrast to previous chemotherapy reporting significantly worse survival for patients with FLT3-ITD, use of FLT3 inhibitors seems to improve their long-term outcome, although there is no definitive evidence showing that their survival can be equal to or even better than those with negative FLT3-ITD.

Although our results showed that FLT3 inhibitors offered favorable outcomes in FLT3-ITD-positive patients, we cannot conclude that patients with negative FLT3-ITD would not benefit from the use of these compounds. Many initial clinical trials of FLT3 inhibitors were carried out in a mixed population, including FLT3-ITD, FLT3-TKD, and FLT3-WT patients, which allowed the investigators to get safety data and establish whether there existed some signaling pathway to justify the use of FLT3 inhibitors in non-FLT3-mutated patients. In some studies, FLT3-WT AML patients were reported to benefit from the use of these drugs and achieve clinical response when FLT3 inhibitors were used as monotherapy. ${ }^{2,18,29}$ Most FLT3 inhibitors are multi-kinase inhibitors functionally and have different targets. Although FLT3 has been shown to be a key mediator of leukemic cell growth and survival, a great number of related and parallel signal transduction pathways contribute to leukemic progression, many of which are likely attenuated by FLT3 inhibitors. ${ }^{44}$ However, the relative nonspecificity may also contribute to the adverse effects associated with these drugs, which may limit their clinical utility. 
Besides the FLT3 status, several other factors might impact the outcome of AML patients with FLT3 inhibitor treatment. Combined use of FLT3 inhibitors with chemotherapy might improve the clinical outcome, which is also seen in the sorafenib subgroup. From five studies ${ }^{11,15,19,25,27}$ focusing sorafenib on R/R AML, the ORR was $18.2 \%$ and $38.5 \%$ in monotherapy and combined therapy, respectively. All the completed large-size RCTs $\mathrm{s}^{33-37}$ added FLT3 inhibitors to conventional chemotherapy, and combined use of sorafenib with chemotherapy in patients with $\mathrm{R} / \mathrm{R}$ AML disease was recommended from the NCCN guidelines. Both the pharmacological action and the better physical status in those who can tolerate chemotherapy might contribute to this fact. It has been reported that FLT3 inhibitors are less effective in the relapsed patients compared with the newly diagnosed patients, which is also seen in our analysis. ${ }^{53,54}$ In Part I, both lestaurtinib studies focused on the addition of lestaurtinib in FLT3-mutant AML patients. The ORR was higher in primary AML than that in relapsed AML $(92.3 \%$ vs $23.8 \%$ in the lestaurtinib group; $94.0 \%$ vs $18.9 \%$ in the control group). In Part II, six out of 21 studies included only primary AML, and patients from these six studies had higher CR (58.3\% vs $7.26 \%$ ) and ORR (65.0\% vs $47.4 \%)$. The dramatic increase in the FLT3 ligand level after chemotherapy represents a possible obstacle to inhibiting FLT3 ${ }^{54}$ Prior therapies might be a key predictor for R/R AML. Ohanian et $\mathrm{al}^{50}$ reported that using crenolanib as a single agent, a much longer median OS was observed in patients with $\leq 2$ prior therapies ( 259 days) than in those with $\geq 3$ prior therapies (53 days). Randhawa et $\mathrm{al}^{49}$ also found that patients without prior FLT3 inhibitor therapy had better CRi, OS, and EFS. Current studies seem to suggest that old patients, especially those older than 60 years, may have worse prognosis. Two RCTs ${ }^{33,36}$ reported the opposite conclusion with sorafenib, saying that patients $\leq 60$ years benefitted from the use of sorafenib with improved EFS and RFS. For R/R AML using crenolanib as a single agent, patients $<60$ years had a higher OS than those $\geq 60$ years (234 vs 185 days). ${ }^{48}$ It has been documented that AML in the elderly is genetically heterogeneous and often follows an MDS. However, Perl et $\mathrm{al}^{55}$ reported that patients aged 70 years with chemotherapy-resistant AML also preserved high response rates and promising survival to quizartinib.

FLT3 inhibitor therapy is associated with an incidence of several adverse effects in clinical trials. Although various drug-related events have been detected, including gastrointestinal symptoms, cardiac toxicity, infections, fatigue, peripheral edema, mucositis, depression, neuropathy, arthralgia, and skin toxicity, ${ }^{15,21}$ FLT3 inhibitors were generally safe and well tolerated in these Phase I or II clinical trials. ${ }^{17,18,20,23,26}$ The most frequent treatment-related events were grades 1 and 2, which were manageable through treatment interruptions, dose reductions, and medical intervention. ${ }^{14}$ Nevertheless, compared with placebo, FLT3 inhibitors might increase the incidence of adverse effects when they were combined with conventional chemotherapy. Two RCTs showed that the addition of sorafenib might lead to more premature termination of study treatment and less consolidation chemotherapy. ${ }^{33,36}$ Similar results were seen in lestaurtinib; the AML15 study showed that there were moderate increases in nausea and diarrhea with the addition of lestaurtinib treatment. ${ }^{34}$ Levis et a ${ }^{35}$ reported that $24 \%$ of patients receiving combined lestaurtinib therapy discontinued their planned therapy before completion because of adverse events. While for midostaurin, Stone et $\mathrm{al}^{37}$ found that there was no significant difference on grades 3 or 4 therapy-related adverse effects compared with placebo. Therefore, high attention should be paid to the treatmentrelated events when FLT3 inhibitors are used in AML.

Several limitations in our analysis should be mentioned. First, only five studies with three types of FLT3 inhibitors were included in Part I. Although the heterogeneity is acceptable, the agent types, age of participants, either monotherapy or combined therapy and AML status including no prior treatment or relapse are not the same between the studies. Therefore, clinical decisions should be made by taking into account multiple factors. Second, the definition of EFS is similar in the studies of Stone et $\mathrm{al}^{37}$ and Röllig et al ${ }^{36}$ as primary treatment failure or relapse or death. However, we could not find the exact definition of EFS from the study of Serve et al. ${ }^{33}$ This may bring potential bias to our study. Third, due to limited patients included and various responses to different FLT3 inhibitor therapies, results of these studies were inconclusive, leading to relatively large CIs of our pooled analysis. More high-quality studies are needed to validate our conclusions. Finally, we pooled ORR including $\mathrm{CR}, \mathrm{CRi}$, and CRp in Part I, and pooled ORR also included PR in Part II. This difference makes it inappropriate to make direct comparison between the data in Part I and those in Part II. Most of the 21 studies in Part II lacked long-term survival data such as OS, EFS, and RFS, and therefore, it is inappropriate to pool them together.

\section{Conclusion}

In this meta-analysis, we showed that addition of FLT3 inhibitors to conventional chemotherapy seems to improve the OS of AML patients. FLT3-ITD-positive patients might be more sensitive to FLT3 inhibitors with a higher CR and 
ORR. FLT3 inhibitors could still give hope to patients who are not candidates for conventional chemotherapy. Based on these findings, we recommend the initiation of more studies to confirm the value of FLT3 inhibitors in AML therapy.

\section{Author contributions}

All authors contributed to the data analysis and drafting and critically revising the paper, gave final approval of the version to be published, and agree to be accountable for all aspects of the work.

\section{Disclosure}

The authors report no conflicts of interest in this work.

\section{References}

1. Percival ME, Lai C, Estey E, Hourigan CS. Bone marrow evaluation for diagnosis and monitoring of acute myeloid leukemia. Blood Rev. 2017;31(4):185-192.

2. Yu MG, Zheng HY. Acute myeloid leukemia: advancements in diagnosis and treatment. Chin Med J (Engl). 2017;130(2):211-218.

3. Smith CC, Wang Q, Chin CS, et al. Validation of ITD mutations in FLT3 as a therapeutic target in human acute myeloid leukaemia. Nature. 2012;485(7397):260-263.

4. Rebechi MT, Pratz KW. Genomic instability is a principle pathologic feature of FLT3 ITD kinase activity in acute myeloid leukemia leading to clonal evolution and disease progression. Leuk Lymphoma. 2017;58(9):1-11.

5. Estey EH. Acute myeloid leukemia: 2013 update on risk-stratification and management. Am J Hematol. 2013;88(4):318-327.

6. Port M, Bottcher M, Thol F, et al. Prognostic significance of FLT3 internal tandem duplication, nucleophosmin 1, and CEBPA gene mutations for acute myeloid leukemia patients with normal karyotype and younger than 60 years: a systematic review and meta-analysis. Ann Hematol. 2014;93(8):1279-1286.

7. Azari-Yam A, Tavakkoly-Bazzaz J, Semnani Y, et al. FLT3 gene mutation profile and prognosis in adult acute myeloid leukemia. Clin Lab. 2016;62(10):2011-2017.

8. Hassanein M, Almahayni MH, Ahmed SO, Gaballa S, El FR. FLT3 inhibitors for treating acute myeloid leukemia. Clin Lymphoma Myeloma Leuk. 2016;16(10):543-549.

9. Hu S, Niu H, Minkin P, et al. Comparison of antitumor effects of multitargeted tyrosine kinase inhibitors in acute myelogenous leukemia. Mol Cancer Ther. 2008;7(5):1110-1120.

10. Hospital MA, Green AS, Maciel TT, et al. FLT3 inhibitors: clinical potential in acute myeloid leukemia. Onco Targets Ther. 2017;10: 607-615.

11. Pratz KW, Cho E, Levis MJ, et al. A pharmacodynamic study of sorafenib in patients with relapsed and refractory acute leukemias. Leukemia. 2010;24(8):1437-1444.

12. Alvarado Y, Kantarjian HM, Luthra R, et al. Treatment with FLT3 inhibitor in patients with FLT3-mutated acute myeloid leukemia is associated with development of secondary FLT3-tyrosine kinase domain mutations. Cancer. 2014;120(14):2142-2149.

13. Cooper TM, Cassar J, Eckroth E, et al. A phase I study of quizartinib combined with chemotherapy in relapsed childhood leukemia: a therapeutic advances in childhood leukemia \& lymphoma (TACL) study. Clin Cancer Res. 2016;22(16):4014-4022.

14. Cortes JE, Kantarjian H, Foran JM, et al. Phase I study of quizartinib administered daily to patients with relapsed or refractory acute myeloid leukemia irrespective of FMS-like tyrosine kinase 3-internal tandem duplication status. J Clin Oncol. 2013;31(29):3681-3687.
15. Crump M, Hedley D, Kamel-Reid S, et al. A randomized phase I clinical and biologic study of two schedules of sorafenib in patients with myelodysplastic syndrome or acute myeloid leukemia: a NCIC (National Cancer Institute of Canada) Clinical Trials Group Study. Leuk Lymphoma. 2010;51(2):252-260.

16. Fiedler W, Kayser S, Kebenko M, et al. A phase I/II study of sunitinib and intensive chemotherapy in patients over 60 years of age with acute myeloid leukaemia and activating FLT3 mutations. Br J Haematol. 2015;169(5):694-700.

17. Fiedler W, Serve H, Dohner H, et al. A phase 1 study of SU11248 in the treatment of patients with refractory or resistant acute myeloid leukemia (AML) or not amenable to conventional therapy for the disease. Blood. 2005;105(3):986-993.

18. Fischer T, Stone RM, Deangelo DJ, et al. Phase IIB trial of oral midostaurin (PKC412), the FMS-like tyrosine kinase 3 receptor (FLT3) and multi-targeted kinase inhibitor, in patients with acute myeloid leukemia and high-risk myelodysplastic syndrome with either wild-type or mutated FLT3. J Clin Oncol. 2010;28(28):4339-4345.

19. Inaba H, Rubnitz JE, Coustan-Smith E, et al. Phase I pharmacokinetic and pharmacodynamic study of the multikinase inhibitor sorafenib in combination with clofarabine and cytarabine in pediatric relapsed/ refractory leukemia. J Clin Oncol. 2011;29(24):3293-3300.

20. Knapper S, Burnett AK, Littlewood T, et al. A phase 2 trial of the FLT3 inhibitor lestaurtinib (CEP701) as first-line treatment for older patients with acute myeloid leukemia not considered fit for intensive chemotherapy. Blood. 2006;108(10):3262-3270.

21. Macdonald DA, Assouline SE, Brandwein J, et al. A phase I/II study of sorafenib in combination with low dose cytarabine in elderly patients with acute myeloid leukemia or high-risk myelodysplastic syndrome from the National Cancer Institute of Canada Clinical Trials Group: trial IND186. Leuk Lymphoma. 2013;54(4):760-766.

22. Ramsingh G, Westervelt P, McBride A, et al. Phase I study of cladribine, cytarabine, granulocyte colony stimulating factor (CLAG regimen) and midostaurin and all-trans retinoic acid in relapsed/refractory AML. Int J Hematol. 2014;99(3):272-278.

23. Ravandi F, Arana YC, Cortes JE, et al. Final report of phase II study of sorafenib, cytarabine and idarubicin for initial therapy in younger patients with acute myeloid leukemia. Leukemia. 2014;28(7):1543-1545.

24. Ravandi F, Cortes JE, Jones D, et al. Phase I/II study of combination therapy with sorafenib, idarubicin, and cytarabine in younger patients with acute myeloid leukemia. J Clin Oncol. 2010;28(11):1856-1862.

25. Röllig C, Brandts C, Shaid S, et al. Survey and analysis of the efficacy and prescription pattern of sorafenib in patients with acute myeloid leukemia. Leuk Lymphoma. 2012;53(6):1062-1067.

26. Stone RM, Fischer T, Paquette R, et al. Phase IB study of the FLT3 kinase inhibitor midostaurin with chemotherapy in younger newly diagnosed adult patients with acute myeloid leukemia. Leukemia. 2012;26(9):2061-2068.

27. Zhang W, Konopleva M, Shi YX, et al. Mutant FLT3: a direct target of sorafenib in acute myelogenous leukemia. J Natl Cancer Inst. 2008;100(3):184-198.

28. Walker AR, Wang H, Walsh K, et al. Midostaurin, bortezomib and MEC in relapsed/refractory acute myeloid leukemia. Leuk Lymphoma. 2016;57(9):2100-2108.

29. Levis MJ, Perl AE, Dombret H, et al. Final results of a phase 2 openlabel, monotherapy efficacy and safety study of quizartinib (AC220) in patients with FLT3-ITD positive or negative relapsed/refractory acute myeloid leukemia after second-line chemotherapy or hematopoietic stem cell transplantation. Blood. 2012;120(21):673.

30. Cortes JE, Perl AE, Dombret H, et al. Final results of a phase 2 openlabel, monotherapy efficacy and safety study of quizartinib (AC220) in patients 60 years of age with FLT3 ITD positive or negative relapsed/ refractory acute myeloid leukemia. Blood. 2012;120(21):48.

31. Iyer SP, Jethava Y, Karanes C, Eckardt JR, Collins R. Safety study of salvage chemotherapy high-dose ara-C/mitoxantrone (HAM) and type I FLT3-TKI crenolanib in first relapsed/primary refractory AML. Blood. 2016;128(22):3983-3983. 
32. Fathi AT, Chen YB. The role of FLT3 inhibitors in the treatment of FLT3mutated acute myeloid leukemia. Eur J Haematol. 2017;98(4):330-336.

33. Serve H, Krug U, Wagner R, et al. Sorafenib in combination with intensive chemotherapy in elderly patients with acute myeloid leukemia: results from a randomized, placebo-controlled trial. J Clin Oncol. 2013;31(25):3110-3118.

34. Knapper S, Russell N, Gilkes A, et al. A randomized assessment of adding the kinase inhibitor lestaurtinib to first-line chemotherapy for FLT3-mutated AML. Blood. 2017;129(9):1143-1154.

35. Levis M, Ravandi F, Wang ES, et al. Results from a randomized trial of salvage chemotherapy followed by lestaurtinib for patients with FLT3 mutant AML in first relapse. Blood. 2011;117(12):3294-3301.

36. Röllig C, Serve H, Huttmann A, et al.; Study Alliance Leukaemia. Addition of sorafenib versus placebo to standard therapy in patients aged 60 years or younger with newly diagnosed acute myeloid leukaemia (SORAML): a multicentre, phase 2, randomised controlled trial. Lancet Oncol. 2015;16(16):1691-1699.

37. Stone RM, Mandrekar S, Sanford BL, et al. The multi-kinase inhibitor midostaurin $(\mathrm{M})$ prolongs survival compared with placebo $(\mathrm{P})$ in combination with daunorubicin (D)/cytarabine (C) induction (ind), high-dose $\mathrm{C}$ consolidation (consol), and as maintenance (maint) therapy in newly diagnosed acute myeloid leukemia (AML) patients (pts) age 18-60 with FLT3 mutations (muts): an international prospective randomized (rand) P-controlled double-blind trial (CALGB 10603/RATIFY [alliance]). Blood. 2015;126(23):6.

38. Creutzig U, Kaspers GJ. Revised recommendations of the International Working Group for diagnosis, standardization of response criteria, treatment outcomes, and reporting standards for therapeutic trials in acute myeloid leukemia. J Clin Oncol. 2004;22(16):3432-3433.

39. Stone RM, Mandrekar SJ, Sanford BL, et al. Midostaurin plus chemotherapy for acute myeloid leukemia with a FLT3 mutation. $N$ Engl $J$ Med. 2017;377(5):454-464.

40. Smith CC, Shah NP. The role of kinase inhibitors in the treatment of patients with acute myeloid leukemia. Am Soc Clin Oncol Educ Book. 2013;2013:313-318.

41. Röllig C, Bornhauser M, Thiede C, et al. Long-term prognosis of acute myeloid leukemia according to the new genetic risk classification of the European LeukemiaNet recommendations: evaluation of the proposed reporting system. J Clin Oncol. 2011;29(20):2758-2765.

42. Dohner H, Estey E, Grimwade D, et al. Diagnosis and management of AML in adults: 2017 ELN recommendations from an international expert panel. Blood. 2017;129(4):424-447.
43. Best-Aguilera C, Rodrigo GO, Elizabeth GA, Monserrat RR. Treatment of acute myeloid leukemia with the FLT3 gene mutation. Curr Oncol Rep. 2017;19(3):21.

44. Wander SA, Levis MJ, Fathi AT. The evolving role of FLT3 inhibitors in acute myeloid leukemia: quizartinib and beyond. Ther Adv Hematol. 2014;5(3):65-77.

45. Stone RM, DeAngelo DJ, Klimek V, et al. Patients with acute myeloid leukemia and an activating mutation in FLT3 respond to a small-molecule FLT3 tyrosine kinase inhibitor, PKC412. Blood. 2005;105(1):54-60.

46. Döhner H, Perl A, Rousselot P, et al. Efficacy and safety of quizartinib (AC220) in patients age $\geq 60$ years with FLT3-ITD-positive relapsed/ refractory acute myeloid leukemia (AML). Haematologica. 2013;98:233.

47. Levis MJ, Martinelli G, Perl AE, et al. The benefit of treatment with quizartinib and subsequent bridging to HSCT for FLT3-ITD $(+)$ patients with AML. J Clin Oncol. 2014;32(15_suppl):7093.

48. Cortes JE, Kantarjian HM, Kadia TM, et al. Crenolanib besylate, a type I pan-FLT3 inhibitor, to demonstrate clinical activity in multiply relapsed FLT3-ITD and D835 AML. J Clin Oncol. 2016;34(15_suppl):7008.

49. Randhawa JK, Kantarjian HM, Borthakur G, et al. Results of a phase II study of crenolanib in relapsed/refractory acute myeloid leukemia patients (Pts) with activating FLT3 mutations. Blood. 2014;124(21):389.

50. Ohanian M, Kantarjian HM, Borthakur G, et al. Efficacy of a type I FLT3 inhibitor, crenolanib, with idarubicin and high-dose ara-C in multiply relapsed/refractory FLT3+ AML. Blood. 2016;128(22):2744.

51. Levis M, Cortes J, Perl A, et al. High response rate and bridging to hematopoietic stem cell transplantation with quizartinib (AC220) in patients with FLT3-ITD-positive relapsed/refractory acute myeloid leukemia (AML). Haematologica. 2013;98:17.

52. Faderl S, Wetzler M, Rizzieri D, et al. Clofarabine plus cytarabine compared with cytarabine alone in older patients with relapsed or refractory acute myelogenous leukemia: results from the CLASSIC I trial. J Clin Oncol. 2012;30(20):2492-2499.

53. Ramos NR, Mo CC, Karp JE, Hourigan CS. Current approaches in the treatment of relapsed and refractory acute myeloid leukemia. J Clin Med. 2015;4(4):665-695.

54. Sato T, Yang X, Knapper S, et al. FLT3 ligand impedes the efficacy of FLT3 inhibitors in vitro and in vivo. Blood. 2011;117(12):3286-3293.

55. Perl AE, Dohner H, Rousselot PH, et al. Efficacy and safety of quizartinib (AC220) in patients age 70 years with FLT3-ITD positive or negative relapsed/refractory acute myeloid leukemia (AML). $J$ Clin Oncol. 2013;31(15_suppl):7023. 


\section{Supplementary materials}
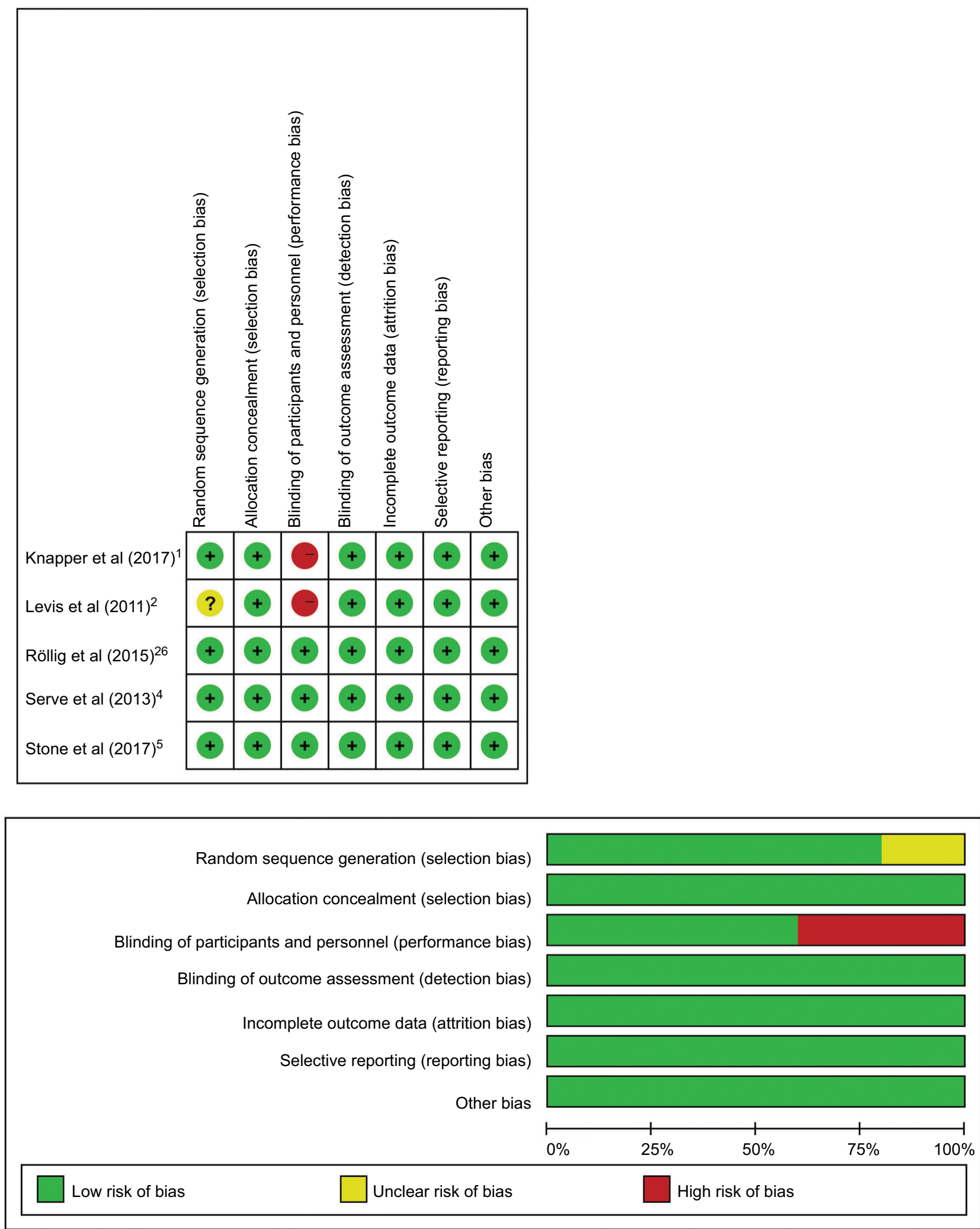

Figure SI Risk of bias graph for five RCTs in Part I.

Abbreviation: RCT, randomized controlled trial. 
A

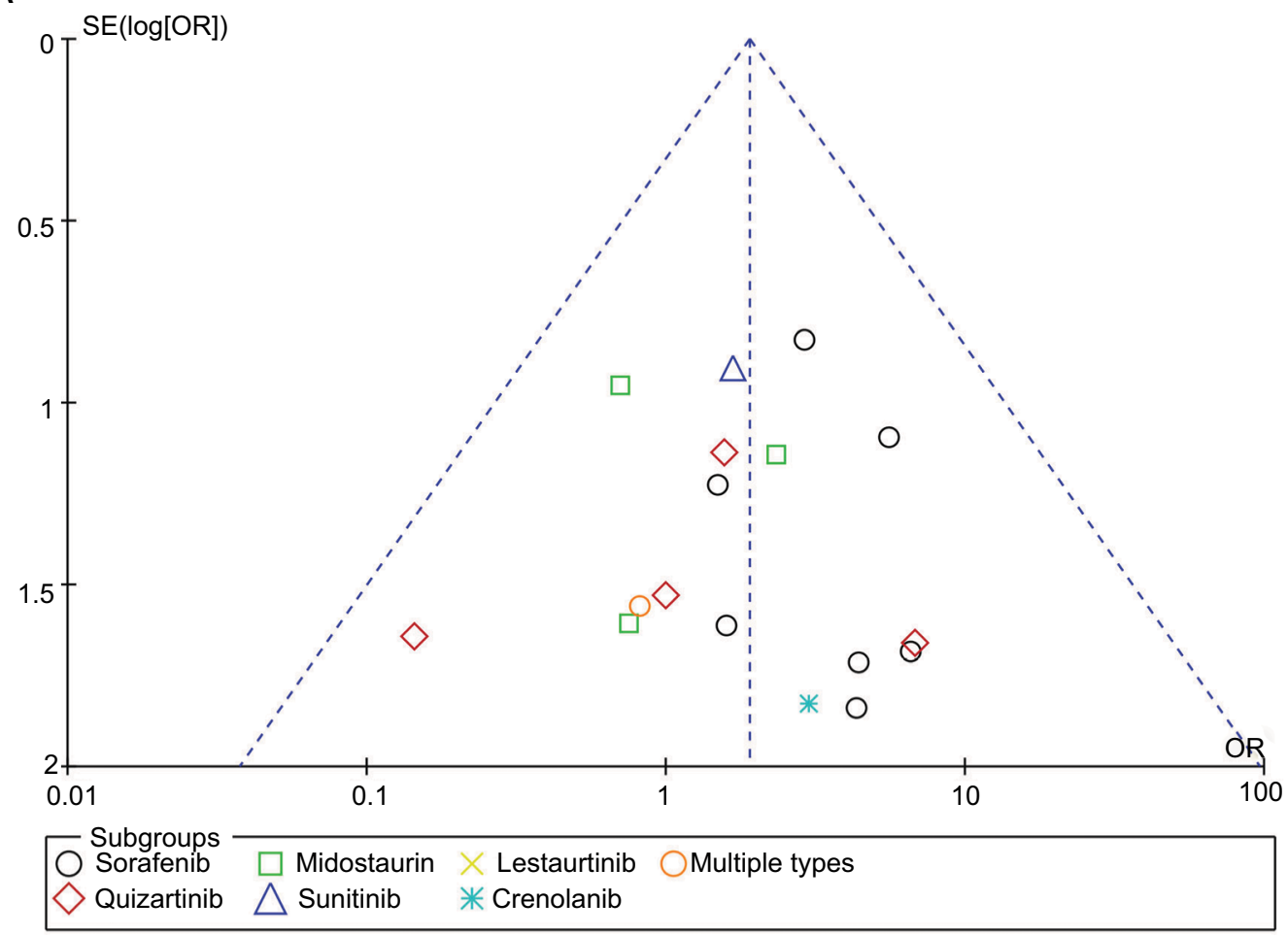

B

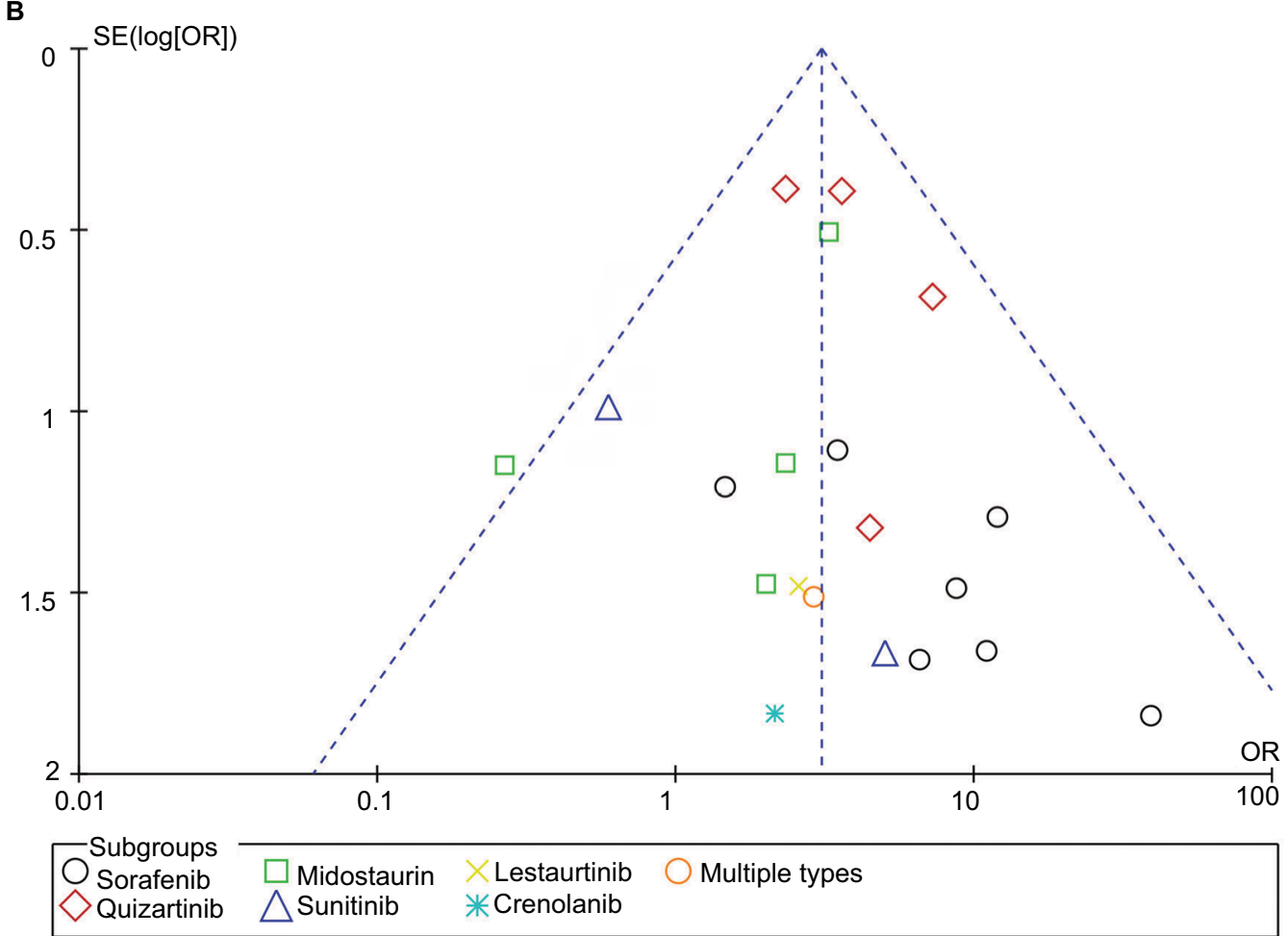

Figure S2 Funnel plot for CR rate (A) and ORR (B) compared between FLT3-ITD positive and negative patients.

Abbreviations: CR, complete remission; ORR, overall response rate; FLT3, FMS-like tyrosine kinase 3; ITD, internal tandem duplication; SE, standard error; OR, odds ratio. 
Table SI Assessment of methodological quality of cohort studies

\begin{tabular}{|c|c|c|c|c|c|c|c|c|c|}
\hline \multirow{2}{*}{$\begin{array}{l}\text { Study } \\
\text { (reference) }\end{array}$} & \multicolumn{4}{|l|}{ Selection } & \multirow[t]{2}{*}{ Comparability } & \multicolumn{3}{|l|}{ Outcome } & \multirow[t]{2}{*}{ Score } \\
\hline & $\begin{array}{l}\text { Representa- } \\
\text { tiveness } \\
\text { of exposed } \\
\text { cohort }\end{array}$ & $\begin{array}{l}\text { Selection of } \\
\text { nonexposed } \\
\text { cohort }\end{array}$ & $\begin{array}{l}\text { Ascertainment } \\
\text { of exposure }\end{array}$ & $\begin{array}{l}\text { Outcome of } \\
\text { interest not } \\
\text { present at } \\
\text { start }\end{array}$ & & $\begin{array}{l}\text { Assessment } \\
\text { of outcome }\end{array}$ & $\begin{array}{l}\text { Follow- } \\
\text { up } \\
\text { length }\end{array}$ & $\begin{array}{l}\text { Follow- } \\
\text { up } \\
\text { adequacy }\end{array}$ & \\
\hline Alvarado et $\mathrm{al}^{6}$ & $*$ & $*$ & $*$ & & $*$ & $*$ & $*$ & & 6 \\
\hline Cooper et $\mathrm{al}^{7}$ & & $*$ & * & & $* *$ & $*$ & $*$ & & 6 \\
\hline Cortes et $\mathrm{al}^{8}$ & & $*$ & $*$ & & $*$ & $*$ & $*$ & $*$ & 6 \\
\hline Cortes et $\mathrm{al}^{9}$ & & $*$ & * & & $* *$ & $*$ & $*$ & $*$ & 7 \\
\hline Crump et al ${ }^{10}$ & $*$ & $*$ & * & $*$ & $*$ & $*$ & $*$ & & 7 \\
\hline Fiedler et al" & & $*$ & * & & $* *$ & $*$ & $*$ & $*$ & 7 \\
\hline Fiedler et $\mathrm{al}^{12}$ & & $*$ & $*$ & & $* *$ & $*$ & $*$ & $*$ & 7 \\
\hline Fischer et $\mathrm{al}^{13 *}$ & & $*$ & $*$ & & $* *$ & $*$ & $*$ & $*$ & 7 \\
\hline Inaba et al $\left.\right|^{14}$ & & $*$ & * & & $* *$ & $*$ & $*$ & $*$ & 7 \\
\hline Knapper et $\mathrm{al}^{24}$ & & $*$ & $*$ & $*$ & $* *$ & $*$ & $*$ & $*$ & 8 \\
\hline Levis et $\mathrm{al}^{23}$ & & $*$ & * & & $*$ & $*$ & $*$ & $*$ & 6 \\
\hline Macdonald et a $\left.\right|^{15}$ & & $*$ & $*$ & & $* *$ & $*$ & $*$ & $*$ & 7 \\
\hline Pratz et al ${ }^{16}$ & & $*$ & $*$ & & $*$ & $*$ & $*$ & $*$ & 6 \\
\hline Ramsingh et al ${ }^{17}$ & & $*$ & $*$ & & $* *$ & $*$ & $*$ & & 6 \\
\hline Ravandi et al ${ }^{18}$ & & $*$ & $*$ & $*$ & $* *$ & $*$ & $*$ & $*$ & 8 \\
\hline Ravandi et al ${ }^{19}$ & & $*$ & $*$ & $*$ & $* *$ & $*$ & $*$ & $*$ & 8 \\
\hline Stone et $\mathrm{a}^{25}$ & & $*$ & * & $*$ & $* *$ & $*$ & $*$ & $*$ & 8 \\
\hline Walker et $\mathrm{al}^{20}$ & & $*$ & * & $*$ & $* *$ & $*$ & $*$ & & 7 \\
\hline Röllig et $\mathrm{al}^{3}$ & $*$ & $*$ & $*$ & & $* *$ & $*$ & $*$ & & 7 \\
\hline lyer et $\mathrm{al}^{21}$ & & $*$ & $*$ & & $*$ & $*$ & $*$ & $*$ & 6 \\
\hline Zhang et $\mathrm{a}^{22}$ & & $*$ & $*$ & & $*$ & & $*$ & $*$ & 5 \\
\hline
\end{tabular}

Note: NOS: study can have one point $(*)$ for meeting each criterion, except that comparability can have a maximum of two points $(* *)$.

Abbreviation: NOS, Newcastle-Ottawa Quality Assessment Scale.

\section{References}

1. Knapper S, Russell N, Gilkes A, et al. A randomized assessment of adding the kinase inhibitor lestaurtinib to first-line chemotherapy for FLT3-mutated AML. Blood. 2017;129(9):1143-1154.

2. Levis M, Ravandi F, Wang ES, et al. Results from a randomized trial of salvage chemotherapy followed by lestaurtinib for patients with FLT3 mutant AML in first relapse. Blood. 2011;117(12):3294-3301.

3. Rollig C, Brandts C, Shaid S, et al. Survey and analysis of the efficacy and prescription pattern of sorafenib in patients with acute myeloid leukemia. Leuk Lymphoma. 2012;53(6):1062-1067.

4. Serve H, Krug U, Wagner R, et al. Sorafenib in combination with intensive chemotherapy in elderly patients with acute myeloid leukemia: results from a randomized, placebo-controlled trial. J Clin Oncol. 2013;31(25):3110-3118.

5. Stone RM, Mandrekar SJ, Sanford BL, et al. Midostaurin plus chemotherapy for acute myeloid leukemia with a FLT3 mutation. $N$ Engl J Med. 2017;377(5):454-464.

6. Alvarado Y, Kantarjian HM, Luthra R, et al. Treatment with FLT3 inhibitor in patients with FLT3-mutated acute myeloid leukemia is associated with development of secondary FLT3-tyrosine kinase domain mutations. Cancer. 2014;120(14):2142-2149.

7. Cooper TM, Cassar J, Eckroth E, et al. A phase I study of quizartinib combined with chemotherapy in relapsed childhood leukemia: a therapeutic advances in childhood leukemia \& lymphoma (TACL) study. Clin Cancer Res. 2016;22(16):4014-4022.

8. Cortes JE, Perl AE, Dombret H, et al. Final results of a phase 2 open-label, monotherapy efficacy and safety study of quizartinib (AC220) in patients 60 years of age with FLT3 ITD positive or negative relapsed/refractory acute myeloid leukemia. Blood. 2012;120 (21):48.
9. Cortes JE, Kantarjian H, Foran JM, et al. Phase I study of quizartinib administered daily to patients with relapsed or refractory acute myeloid leukemia irrespective of FMS-like tyrosine kinase 3-internal tandem duplication status. J Clin Oncol. 2013;31(29):3681-3687.

10. Crump M, Hedley D, Kamel-Reid S, et al. A randomized phase I clinical and biologic study of two schedules of sorafenib in patients with myelodysplastic syndrome or acute myeloid leukemia: a NCIC (National Cancer Institute of Canada) Clinical Trials Group Study. Leuk Lymphoma. 2010;51(2):252-260.

11. Fiedler W, Serve H, Dohner H, et al. A phase 1 study of SU11248 in the treatment of patients with refractory or resistant acute myeloid leukemia (AML) or not amenable to conventional therapy for the disease. Blood. 2005;105(3):986-993.

12. Fiedler W, Kayser S, Kebenko M, et al. A phase I/II study of sunitinib and intensive chemotherapy in patients over 60 years of age with acute myeloid leukaemia and activating FLT3 mutations. $\mathrm{Br} J$ Haematol. 2015;169(5):694-700.

13. Fischer T, Stone RM, Deangelo DJ, et al. Phase IIB trial of oral midostaurin (PKC412), the FMS-like tyrosine kinase 3 receptor (FLT3) and multi-targeted kinase inhibitor, in patients with acute myeloid leukemia and high-risk myelodysplastic syndrome with either wild-type or mutated FLT3. J Clin Oncol. 2010;28(28):4339-4345.

14. Inaba H, Rubnitz JE, Coustan-Smith E, et al. Phase I pharmacokinetic and pharmacodynamic study of the multikinase inhibitor sorafenib in combination with clofarabine and cytarabine in pediatric relapsed/ refractory leukemia. J Clin Oncol. 2011;29(24):3293-3300.

15. Macdonald DA, Assouline SE, Brandwein J, et al. A phase I/II study of sorafenib in combination with low dose cytarabine in elderly patients with acute myeloid leukemia or high-risk myelodysplastic syndrome from the National Cancer Institute of Canada Clinical Trials Group: trial IND186. Leuk Lymphoma. 2013;54(4):760-766. 
16. Pratz KW, Cho E, Levis MJ, et al. A pharmacodynamic study of sorafenib in patients with relapsed and refractory acute leukemias. Leukemia. 2010;24(8):1437-1444.

17. Ramsingh G, Westervelt P, McBride A, et al. Phase I study of cladribine, cytarabine, granulocyte colony stimulating factor (CLAG regimen) and midostaurin and all-trans retinoic acid in relapsed/refractory AML. Int J Hematol. 2014;99(3):272-278.

18. Ravandi F, Cortes JE, Jones D, et al. Phase I/II study of combination therapy with sorafenib, idarubicin, and cytarabine in younger patients with acute myeloid leukemia. J Clin Oncol. 2010;28(11):1856-1862.

19. Ravandi F, Arana YC, Cortes JE, et al. Final report of phase II study of sorafenib, cytarabine and idarubicin for initial therapy in younger patients with acute myeloid leukemia. Leukemia. 2014;28(7):1543-1545.

20. Walker AR, Wang H, Walsh K, et al. Midostaurin, bortezomib and MEC in relapsed/refractory acute myeloid leukemia. Leuk Lymphoma. 2016;57(9):2100-2108.

21. Iyer SP, Jethava Y, Karanes C, Eckardt JR, Collins R. Safety study of salvage chemotherapy high-dose ara-C/mitoxantrone (HAM) and type I FLT3-TKI crenolanib in first relapsed/primary refractory AML. Blood. 2016;128(22):3983-3983.
22. Zhang W, Konopleva M, Shi YX, et al. Mutant FLT3: a direct target of sorafenib in acute myelogenous leukemia. J Natl Cancer Inst. 2008;100(3):184-198.

23. Levis MJ, Perl AE, Dombret H, et al. Final results of a phase 2 openlabel, monotherapy efficacy and safety study of quizartinib (AC220) in patients with FLT3-ITD positive or negative relapsed/refractory acute myeloid leukemia after second-line chemotherapy or hematopoietic stem cell transplantation. Blood. 2012;120(21):673.

24. Knapper S, Burnett AK, Littlewood T, et al. A phase 2 trial of the FLT3 inhibitor lestaurtinib (CEP701) as first-line treatment for older patients with acute myeloid leukemia not considered fit for intensive chemotherapy. Blood. 2006;108(10):3262-3270.

25. Stone RM, Fischer T, Paquette R, et al. Phase IB study of the FLT3 kinase inhibitor midostaurin with chemotherapy in younger newly diagnosed adult patients with acute myeloid leukemia. Leukemia. 2012;26(9):2061-2068.

26. Röllig C, Serve H, Huttmann A, et al.; Study Alliance Leukaemia. Addition of sorafenib versus placebo to standard therapy in patients aged 60 years or younger with newly diagnosed acute myeloid leukaemia(SORAML): a multicentre, phase 2, randomised controlled trial. Lancet Oncol. 2015;16(16):1691-1699.
Cancer Management and Research

\section{Publish your work in this journal}

Cancer Management and Research is an international, peer-reviewed open access journal focusing on cancer research and the optimal use of preventative and integrated treatment interventions to achieve improved outcomes, enhanced survival and quality of life for the cancer patient. The manuscript management system is completely online and includes
Dovepress

a very quick and fair peer-review system, which is all easy to use. Visit http://www.dovepress.com/testimonials.php to read real quotes from published authors. 